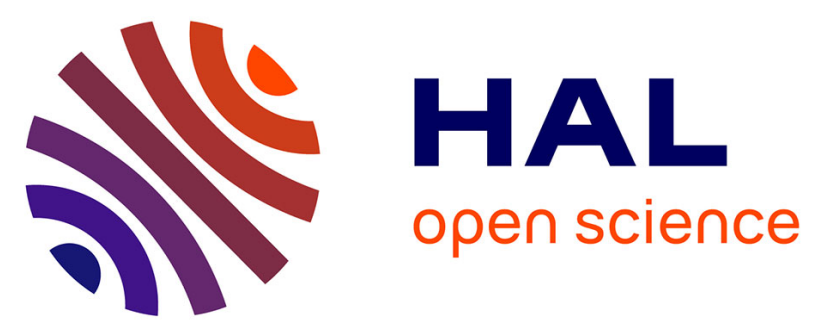

\title{
Carrier Frequency Offset Estimation Based on Circular Harmonic Expansion for Optical Coherent M-QAM Communication Systems
}

Trung Hien Nguyen, Michel Joindot, Mathilde Gay, Laurent Bramerie, Jean-Claude Simon, Pascal Scalart, Olivier Sentieys

\section{To cite this version:}

Trung Hien Nguyen, Michel Joindot, Mathilde Gay, Laurent Bramerie, Jean-Claude Simon, et al.. Carrier Frequency Offset Estimation Based on Circular Harmonic Expansion for Optical Coherent M-QAM Communication Systems. 20th Opto-Electronics and Communications Conference (OECC 2015), Jun 2015, Shanghai, China. pp.1-3, 10.1109/OECC.2015.7340175 . hal-01316911

\author{
HAL Id: hal-01316911 \\ https://hal.science/hal-01316911
}

Submitted on 17 May 2016

HAL is a multi-disciplinary open access archive for the deposit and dissemination of scientific research documents, whether they are published or not. The documents may come from teaching and research institutions in France or abroad, or from public or private research centers.
L'archive ouverte pluridisciplinaire HAL, est destinée au dépôt et à la diffusion de documents scientifiques de niveau recherche, publiés ou non, émanant des établissements d'enseignement et de recherche français ou étrangers, des laboratoires publics ou privés. 


\section{Carrier Frequency Offset Estimation Based on Circular Harmonic Expansion for Optical Coherent $M-\mathrm{QAM}$ Communication Systems}

\author{
Trung-Hien Nguyen, Michel Joindot, Mathilde Gay, \\ Laurent Bramerie, Jean-Claude Simon \\ FOTON Laboratory, CNRS, University of Rennes 1, \\ ENSSAT, F-22305 Lannion, France \\ trung-hien.nguyen@enssat.fr
}

\author{
Pascal Scalart, Olivier Sentieys \\ INRIA / IRISA, Campus Beaulieu \\ F-35000 Rennes, France \\ pascal.scalart@enssat.fr
}

\begin{abstract}
Circular harmonic expansion-based carrier frequency offset estimation is investigated for optical $M-$ QAM communication systems. The proposed method, combined with a gradient-descent algorithm, shows better performance compared to already proposed VVMFOE and $4^{\text {th }}$ power methods.
\end{abstract}

Keywords-M-QAM; optical communication; coherent communication; carrier frequency offset.

\section{INTRODUCTION}

Coherent detection and $M$-ary quadrature amplitude modulation ( $M$-QAM) signals are very attractive for optical transmission systems beyond $100 \mathrm{Gbit} / \mathrm{s}$ [1]. Coherent detection is usually based on the intradyne detection scheme with unlocked transmitter (Tx) and local oscillator (LO) lasers. In such a scheme, the carrier frequency offset (CFO) between the Tx and $\mathrm{LO}$ can reach values as high as a few $\mathrm{GHz}$ [2], which does not allow proper recovery of the signal if not compensated. Frequency offset estimation (FOE) is hence required in the digital signal processing (DSP) at the receiver (Rx). Substantial efforts have been dedicated to the design of FOE solutions that do not require training symbols in either feedback [1] or feedforward [3] manners and using time domain [4] or frequency domain [5] approaches. Among those, feedforward FOE provides a better laser phase noise tolerance and is more hardware-efficient than feedback FOE [6]. Moreover, the use of periodograms in frequency domain analysis can quickly estimate the CFO with a smaller number of required symbols compared to the time domain approach [5].

The well-known Viterbi-Viterbi fourth power estimator (VV4PE) used for the FOE of $M$-ary phase shift keying ( $M$-PSK) signals [3] is no longer valid for $M$-QAM, especially for cross $M$-QAM signals (e.g. 32-QAM). Several feedforward FOE methods based on the maximization of symbols periodograms using the fast-Fourier transform (FFT) have been proposed so far [5], including a solution for widening the FOE range [7]. However, the applicability of these methods to cross $M$-QAM was not discussed in detail. Recently, the Viterbi-Viterbi monomial FOE (VVMFOE) method has been reported in [8], with an extension for cross $M$-QAM constellations. The idea behind this method is to assign weight coefficients to symbols with different amplitude levels, usually with negative power for cross $M$-QAM signals. However, the choice of this negative power value is empirical and the negative power may make the implementation in field programmable gate arrays (FPGAs) more complex and require more computational efforts.

In this paper, we investigate an FOE technique based on the circular harmonic expansion (CHE) of the log-likelihood function (LLF) for both square and cross $M$-QAM signals, called CHEFOE. Based on this expansion, only the first significant term of the resultant series is chosen as an optimum weighting function for FOE. In this way, a look-up table (LUT) can be easily implemented in FPGAs for weighting the symbol amplitudes. This implementation reduces the complexity since it does not make use of negative power. Although FOE based on the CHE method has been presented in [9], it has, to the best of our knowledge, never been exploited in optical coherent communication systems, in which the typical linewidths of commercial distributed feedback (DFB) and external cavity lasers (ECL) range from several $\mathrm{kHz}$ to the order of $10 \mathrm{MHz}$, potentially leading to significant phase noise in the received $M-\mathrm{QAM}$ constellation. Moreover, the combination of CHEFOE and gradient-descent algorithm [5] is also proposed and studied. This proposal provides more accurate estimation of CFO with small mean square error (MSE) and smaller required number of symbols, showing the effectiveness of the proposed method.

\section{CIRCUlAR HARMONIC EXPANSIONS OF THE MAXIMUM LOGLIKELIHOOD FUNCTION FOR CFO ESTIMATION}

In previous work $[5,7] \quad\left(4^{\text {th }}\right.$ power method $)$, the maximization of the following periodogram of the received QAM signal, $x(k)=r(k) \cdot \exp (j \cdot \phi(k))$, is used to estimate the CFO value, $\phi_{f}=2 \pi \cdot \Delta f \cdot T_{B}$

$$
\widehat{\phi_{f}}=\frac{1}{4} \arg \max _{\varphi \in[-1 / 2,1 / 2)} \sum_{p=1}^{2}\left|\frac{1}{N} \sum_{k=0}^{N-1} x^{4}(k) e^{-j 2 \pi k \varphi}\right|^{2}
$$


where $T_{B}$ is the symbol period, $N$ denotes the number of symbols and $p$ index the polarization. For simplicity, the following study considers a unique periodogram associated with a single polarization.

Assuming the received symbols at the baud rate are corrupted by complex additive white Gaussian noise (AWGN) with equal variance $\sigma^{2}$ for both real and imaginary parts, the LLF function for an unknown phase shift due to CFO and the laser phase noise, $\phi_{0}$, is represented as follows [9]

$$
\operatorname{LLF}(\phi(k) \mid x(k))=\log \left[\frac{1}{2 \pi \sigma^{2} M} \sum_{m=1}^{M} \exp \left(-\frac{\left|x(k) e^{-j \phi(k)}-C_{m}\right|^{2}}{2 \sigma^{2}}\right)\right]
$$

where the $C_{m}$ denote the ideal values of the constellation symbols, $m=1, \ldots, M$, and $\phi(k)=\phi_{0}+k \cdot \phi_{f}$.

Expanding (2) in Fourier series along the phase $\varphi$, the LLF approximation retaining only the first nonzero harmonic component over $N$ symbols is given by [9]

$$
\operatorname{LLF}\left(\phi_{0}, \phi_{f} \mid x(k)\right) \approx \operatorname{Re}\left[e^{-j 4 \phi_{0}} F_{4}\left(4 \phi_{f}\right)\right]
$$

in which $F_{4}\left(4 \phi_{f}\right)=\sum_{k=1}^{N} A_{4}(r(k)) e^{-j 4 \varphi(k)} e^{-j 4 k \phi_{f}}$, and $A_{4}(r)$ is the amplitude of the fourth harmonic. rule

The CFO is compensated based on the following estimator

$$
\widehat{\phi_{f}}=\frac{1}{4} \arg \max _{4 \phi_{f}}\left|F_{4}\left(4 \phi_{f}\right)\right|
$$

Comparing (4) to (1), it can be observed that CHEFOE is another version of the $4^{\text {th }}$ power estimator with radius weighting function and without the (negative) power requirement for signal amplitudes. It is also shown that the VVMPE estimator based on optimal nonlinear least-square (NLLS) is inferior to the CHE estimator, especially for cross $M-\mathrm{QAM}$ constellations, due to the sign-changing nature of the weighting function $A_{4}(k)$ [9].

Fig. 1 presents the configuration for $M$-QAM FOE based on the CHE method. The proposed estimator is cascaded with a fine step implementation of the gradient-descent algorithm to fast converge and to minimize the MSE of the estimated CFO. The fine estimated CFO is given by [5]

$$
\widehat{\phi_{f}}(k+1)=\widehat{\phi_{f}}(k)+\mu\left[\left.\frac{\partial F_{4}\left(4 \phi_{f}\right)}{\partial \phi_{f}}\right|_{\widehat{\phi}_{f}(k)} /\left.\frac{\partial^{2} F_{4}\left(4 \phi_{f}\right)}{\partial \phi_{f}^{2}}\right|_{\widehat{\phi_{f}}(k)}\right]
$$

in which $\mu$ is the step size depending on the symbol block length. This optimization technique reduces the MSE to $1 / N^{3}[5]$.

\section{NUMERICAL RESULTS AND DISCUSSION}

In order to evaluate the performance of the proposed FOE method, numerical simulations are carried out with 10 GBaud

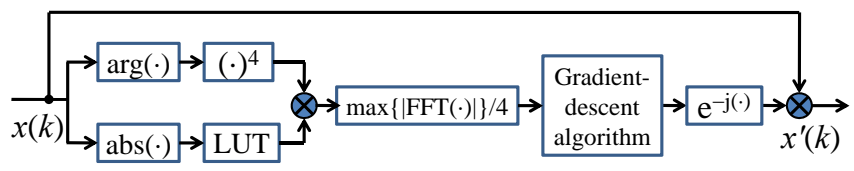

Fig. 1. Block diagram of CHEFOE.

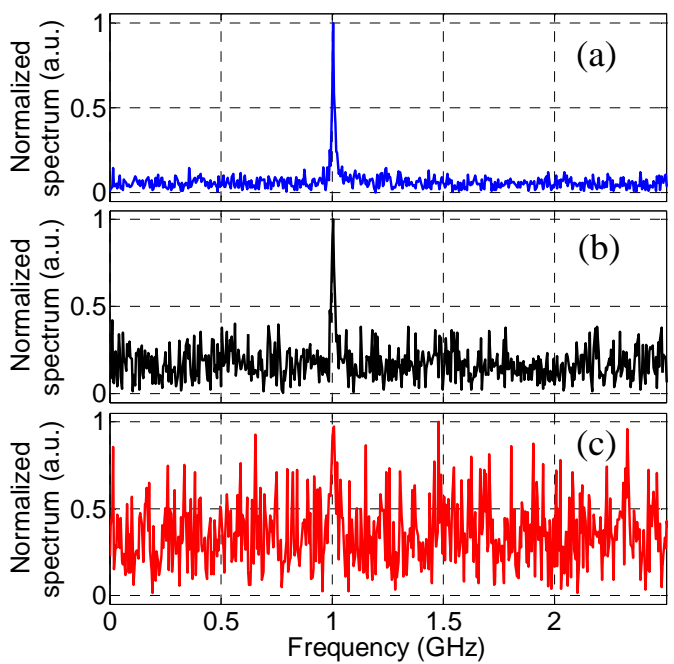

Fig. 2. Examples of normalized spectra of 32-QAM signals with CFO of $0.25 \mathrm{GHz}$ using (a) CHEFOE; (b) VVMFOE; (c) $4^{\text {th }}$ power methods.

16-QAM and 32-QAM signals under the impact of phase noise originating from the laser sources. In the transmitter, the $M$-QAM signals are generated by differential encoding of a pseudo-random binary sequence (PRBS) with length of $2^{13}-1$ onto the constellation. The phase noise caused by the combined laser linewidth, $\Delta v$, is modeled as a discrete time random walk $\phi_{n}=\phi_{n-1}+\Delta_{n}$, in which $\Delta_{n}$ is a Gaussian random variable with zero mean and variance $2 \pi \cdot \Delta v \cdot T_{B}$. Moreover, 130 000 symbols are transmitted and corrupted by AWGN noise. The noise is specified by the signal-to-noise ratio (SNR) in the electrical domain. To focus on the FOE function, other sources of impairments such as chromatic dispersion, polarization mode dispersion and timing clock recovery errors are assumed to be completely compensated. Finally, the received symbols are decimated to the baud rate before being sent to the FOE stage. In the following study, 16-QAM and 32-QAM signals at the respective SNRs of $18.4 \mathrm{~dB}$ and $20.8 \mathrm{~dB}$, corresponding to $1 \mathrm{~dB}$ penalty at the hard forwarderror correction (FEC) BER limit of $10^{-3}$ are used. The mean and standard deviation (STD) of the CFO estimator are calculated based on 500 runs of simulation and compared to the $4^{\text {th }}$ power [5] and VVMFOE [8] methods.

In the first step, the CFO is set to $0.5 \mathrm{GHz}$ and $0.25 \mathrm{GHz}$ for 16-QAM and 32-QAM signals, respectively. The gradient-descent algorithm is not activated for a fair comparison. Fig. 2 presents examples of normalized spectra of 32-QAM signals obtained using 1024 symbols. It can be seen that the CHEFOE and VVMFOE methods provide a better periodogram than the $4^{\text {th }}$ power method. However, the periodogram obtained by CHEFOE is less noisy than that of VVMFOE, indicating a better estimator. In the next step, the CFO estimation error (the difference between the predefined CFO and the estimated one) is evaluated as a function of 


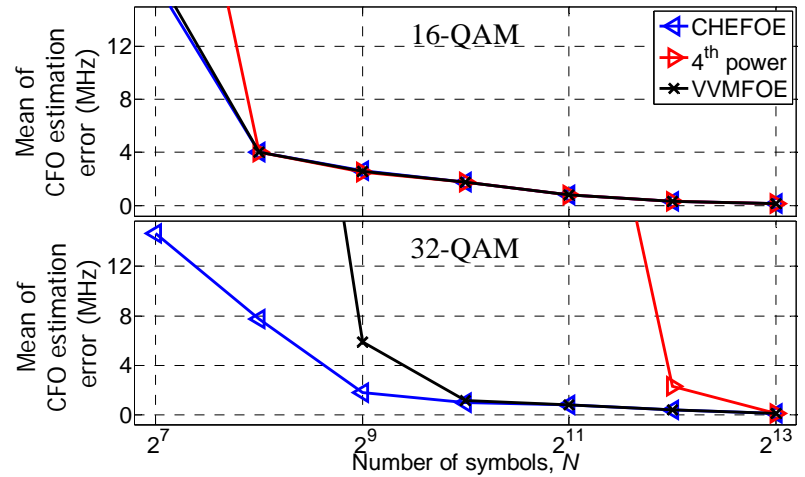

Fig. 3. Average value of the CFO estimation error as a function of the number of symbols, $N$.

number of utilized symbols, $N$. The mean (Fig. 3 ) and the STD (not plotted due to space limitations) of the CFO estimation error are investigated for 16-QAM (upper row) and 32-QAM (lower row) signals. For 16-QAM signals, the three methods give similar performance as the number of symbols exceeds $2^{8}$, resulting in mean and STD of estimation error smaller than $4 \mathrm{MHz}$ and $2 \mathrm{MHz}$, respectively. For 32-QAM signals, the required number of symbols for CHEFOE, VVMFOE and $4^{\text {th }}$ power methods required to achieve similar mean and STD values as for $16-\mathrm{QAM}$ signals are $2^{9}, 2^{10}$ and $2^{13}$, respectively. It means that the proposed method requires less than half the number of symbols for FOE compared to VVMFOE and 8 times less than the $4^{\text {th }}$ power method, indicating the robustness of the CHEFOE method. The laser linewidth tolerance of the three methods is further studied (Fig. 4) with symbol lengths of $2^{9}$ and $2^{10}$ for $16-\mathrm{QAM}$ and 32-QAM signals, respectively. For 16-QAM signals, the normalized linewidths that can be tolerated can take values as high as $2.5 \times 10^{-3}, 10^{-3}$ and $5 \times 10^{-4}$ using the CHEFOE, VVMFOE and $4^{\text {th }}$ power methods, respectively, in order to obtain a mean of the estimation errors inferior to $5 \mathrm{MHz}$. For 32-QAM signals, the $4^{\text {th }}$ power method does not work, whereas the normalized linewidth tolerance of the VVMFOE method reduces to $10^{-4}$. The CHEFOE method still allows a normalized linewidth tolerance up to $2.5 \times 10^{-3}$ for a mean of the estimation error below $5 \mathrm{MHz}$, confirming the effectiveness of the proposed method.

Finally, the gradient-descent algorithm combined with CHEFOE is activated and considered only for 16-QAM signals in comparison to the VVMFOE method with $N=256$. The extrema of periodogram are chosen such that $4 \phi_{f} T_{B}$ lies in two adjacent FFT points. Fig. 5 presents the mean and STD of the estimation error using the CHEFOE and VVMFOE methods before and after applying the gradient-descent algorithm. It can be observed that the combination of FOE with gradient descent algorithm reduces by up to a factor two the CFO estimation error compared to FOE alone, indicating the accuracy of the proposed combination estimator.

\section{CONCLUSIONS}

A carrier frequency offset estimation based on circular harmonic expansion in combination with the gradient-descent algorithm is proposed and numerically studied for optical

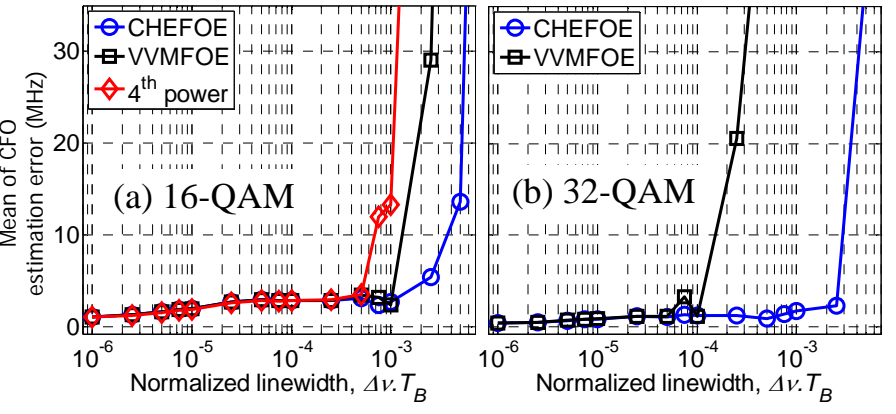

Fig. 4. CFO estimation error versus normalized linewidth.

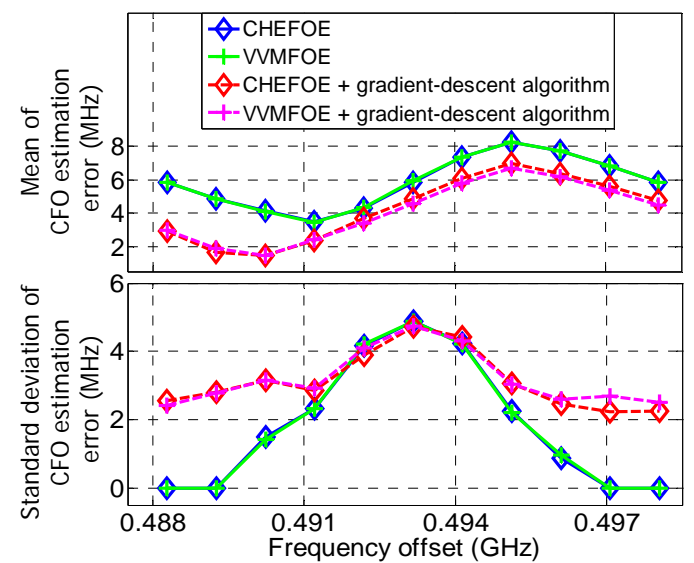

Fig. 5. Mean and STD of the estimation errors for 16-QAM in a FFT interval.

$M$-QAM communication systems. The proposed method outperforms the VVMFOE and $4^{\text {th }}$ power methods in terms of number of required symbols and laser linewidth tolerance, especially for cross $M$-QAM signals.

\section{ACKNOWLEDGMENT}

This work was supported by the French National Research Agency (OCELOT project, ref. ANR-10-VERS-0015), the Contrat de plan EtatRégion Ponant and the French Ministry of Research. We acknowledge Christophe Peucheret for his valuable comments and discussion.

\section{REFERENCES}

[1] P. J. Winzer et al., "Spectrally efficient long-haul optical networking ...,” IEEE J. Lightw. Technol., vol. 28, no. 4, pp. 547-556, Feb. 2010.

[2] M. Funabashi et al., "Recent adddvances in DFB lasers ...,” IEEE J. Sel. Topics Quantum Electron., vol. 10, no. 2, pp. 312-318, Apr. 2004.

[3] A. Leven, et al., "Frequency estimation in intradyne reception," IEEE Photon. Technol. Lett., vol. 19, no. 6, pp. 366-368, Mar. 2007.

[4] L. Li et al., "Wide-range, accurate and simple digital frequency offset compensator ...," in Proc. OFC, paper OWT4, 2008.

[5] M. Selmi et al., "Accurate digital frequency offset estimator for coherent PolMux QAM ...,” in Proc. ECOC, paper P3.08, 2009.

[6] T. Pfau et al., "Hardware-efficient coherent digital receiver concept ...," IEEE J. Lightw. Technol., vol. 27, no. 8, pp. 989-999, Apr. 2009.

[7] Y. Cao et al., "Modified frequency and phase estimation for M-QAM optical coherent detection,” in Proc. ECOC, paper We.7.A.1, 2010.

[8] C. Spatharakis et al., "Frequency offset estimation and carrier phase recovery ...,” in Proc. CSNDSP, pp. 781-786, Jul. 2014.

[9] A. B. Sergienko, and A. V. Petrov, "Blind frequency offset estimation for QAM signals ...,” in Proc. EWDTS, pp. 278-281, 2010. 